\title{
Método de Pontos Interiores Aplicado ao Problema de Fluxo de Potência Ótimo com Demanda Incerta
}

\author{
Demacio Costa de Oliveira ${ }^{1}$ \\ UFRPE, Serra Talhada, PE \\ Aurelio Ribeiro Leite de Oliveira ${ }^{2}$ \\ UNICAMP, Campinas, SP
}

\begin{abstract}
Resumo. O Problema de Fluxo de Potência Ótimo é importante em sistemas de potência, pois pode ser aplicado no despacho econômico, análise de confiabilidade de geração e transmissão, análise de segurança e programação da geração de curto prazo. Contudo, as decisões no setor energético dependem de parâmetros incertos presentes nos planejamentos de curto e longo prazo. Deste modo, propomos um modelo de Programação Estocástica de Dois Estágios com Recurso Fixo para o Problema de Fluxo de Potência Ótimo com Demanda Incerta, o qual é resolvido pelo Método Seguidor de Caminhos, que é um Método de Pontos Interiores. As fontes de geração de potência ativa consideradas foram as usinas hidrelétricas e térmicas. Testes numéricos realizados no problema IEEE30 indicam que seria vantajoso considerar a solução estocástica.
\end{abstract}

Palavras-chave. Método de Pontos Interiores, Programação Estocástica de Dois Estágios com Recurso Fixo, Fluxo de Potência Ótimo.

\section{Introdução}

Fluxo de Potência Ótimo (FPO) é um termo relacionado a uma classe de problemas do sistema energético nos quais se deseja otimizar uma função objetivo sujeita a um conjunto de restrições, que representam limites operacionais e leis físicas da rede elétrica [8]. Neste trabalho, usaremos um modelo de fluxo de redes para o Fluxo de Potência Ótimo CC, o qual tem apresentado resultados satisfatórios nas aplicações [9]. Os dados incertos presentes nos planejamentos de curto e longo prazo do setor energético são classificados como parâmetros técnicos e econômicos. Os parâmetros técnicos são divididos em topológicos e operacionais. Parâmetros topológicos estão relacionados a rede de transmissão, como falhas ou interrupções de linhas, geradores ou dispositivos de medição. Parâmetros operacionais contemplam as decisões referentes a demanda ou ao valor de geração. Parâmetros econômicos são aqueles relacionados a índices econômicos estudados na microeconomia ou macroeconomia. Desse modo, a fim de tratar essas incertezas, surgiram vários métodos de otimização, cuja principal diferença consiste na técnica escolhida para descrever a incerteza dos parâmetros de entrada. Esses métodos são classificados como: abordagem probabilística, possibilística, probabilística-possibilística, teoria de decisão da informação do gap, otimização robusta e análise de intervalo [11].

Um dos primeiros trabalhos sobre programação estocástica foi publicado em 1955 por Dantzig. Segundo ele, a motivação do estudo surgiu de uma proposta de estender os métodos de programação linear para resolver problemas de alocação com demanda incerta. Dantzig afirma que a

\footnotetext{
${ }^{1}$ demacio.oliveira@ufrpe.br.

aurelio@ime.unicamp.br
} 
característica fundamental dos problemas sob incerteza é que as decisões são tomadas em dois ou mais estágios, assim, as variáveis de primeiro estágio são as únicas que podem ser determinadas com antecedência, porque as de segundo estágio e posteriores dependem dos eventos aleatórios [3]. Em geral, resolver programas estocásticos aumenta a complexidade do problema. Assim, na prática, os parâmetros incertos são substituídos por aproximações ou valores esperados e, então, problemas mais simples são resolvidos. Para avaliar a qualidade da solução de um programa estocástico, são usadas algumas medidas. Neste trabalho, destacaremos o Valor da Solução Estocástica (VSS) que avalia o desempenho da solução do modelo determinístico em relação à solução do programa estocástico [1].

Os Métodos de Pontos Interiores (MPIs) surgiram em 1967 com o trabalho de Dikin. Em 1984, Karmarkar apresentou um MPI baseado em geometria projetiva e otimização sobre uma esfera. Este método possui complexidade polinomial e pode ser visto como um aprimoramento do método dos elipsóides [4,6]. Dentre os avanços na classe dos MPIs, destacamos os métodos primaldual. As características destes métodos são: transformar o problema primal num problema de barreira equivalente, aplicar as condições de otimalidade de primeira ordem na função lagrangiana e resolver o sistema de equações não lineares pelo método de Newton. Assim, estes métodos resolvem os problemas primal e dual ao mesmo tempo $[4,7,12]$. Os MPIs são usados na resolução de problemas linear, não linear, convexo e não convexo. Na área de otimização da operação de sistemas de potência os métodos de pontos interiores têm desempenhado papel relevante, devido ao tamanho e características desses problemas [10].

Diante desse contexto, pretendemos investigar a incerteza da demanda, que é um parâmetro operacional, por meio de uma técnica probabilística. Assim, estamos propondo um modelo de programação estocástica de dois estágios com recurso fixo para o FPO com demanda incerta. Desse modo, uma função de distribuição de probabilidade será obtida por meio da análise do histórico de consumo. Em seguida, usaremos uma aproximação discreta da distribuição de probabilidade para obter aproximações do problema contínuo e analisar sua convergência. O Método Seguidor de Caminhos foi escolhido para a resolução desse problema, pois apresenta bons resultados quando aplicado a sistemas de potência $[9,10]$. Por fim, usaremos o parâmetro estocástico VSS para analisar a importância de se considerar a incerteza da demanda no FPO.

\section{Fluxo de Potência Ótimo CC}

O problema de fluxo de potência ótimo CC pode ser formulado por um modelo de fluxo em redes cuja função objetivo resulta da soma de duas funções quadráticas separáveis, uma função representa as perdas no sistema de transmissão, e outra função corresponde aos custos de geração das usinas termelétricas e hidrelétricas. No conjunto de restrições, as leis de Kirchhoff dos nós e ramos, assim como os limites de geração e transmissão, são consideradas $[2,9]$.

$$
\begin{array}{cc}
\text { Minimize } & \frac{\beta}{2}\left(p^{T} Q p+c_{q}^{T} p\right)+\frac{\alpha}{2} f^{T} R f \\
\text { Sujeito a } & -E p+A f=-l \\
& X f=0 \\
& p_{l} \leq p \leq p_{u} \\
& f_{l} \leq f \leq f_{u},
\end{array}
$$

onde $\beta$ e $\alpha$ são escalares usados para especificar o objetivo da otimização. A matriz diagonal $Q \in \mathbb{R}^{g \times g}$ e o vetor $c_{q} \in \mathbb{R}^{g}$ representam os custos de geração. A matriz diagonal $R \in \mathbb{R}^{n \times n}$ corresponde à resistência das linhas de transmissão. A matriz $E \in \mathbb{R}^{m \times g}$ é formada por colunas da matriz identidade, e cada coluna sua representa uma barra de geração. As matrizes $A \in \mathbb{R}^{m \times n}$ e $X \in \mathbb{R}^{n-m+1 \times n}$ correspondem à incidência e reatância da rede de transmissão, respectivamente. 
O vetor $l \in \mathbb{R}^{m}$ denota a demanda de potência ativa. Os vetores $p, p_{l}$ e $p_{u}$, de $\mathbb{R}^{g}$, representam a geração de potência ativa e seus limites inferior e superior, respectivamente. Analogamente, os vetores $f, f_{l}$ e $f_{u}$, de $\mathbb{R}^{n}$, estão relacionadas ao fluxo de potência ativa.

\subsection{Mudança de Variáveis}

A fim de reescrever o problema de FPO na forma padrão, definimos $x=f-f l$ e $g=p-p l$. Além disso, juntamos as equações (1) e (2), definindo $\bar{R}=[R ; 0], \overline{c_{r}}=\left[2 R f_{l} ; 0\right], \overline{x_{u}}=\left[x_{u} ; 0\right]$, $\overline{c_{q}}=c_{q}+2 Q p_{l}$,

$$
B=\left[\begin{array}{c}
A \\
X
\end{array}\right], \bar{B}=\left[\begin{array}{ll}
B & e_{n+1}
\end{array}\right] \text { e } \bar{E}=\left[\begin{array}{c}
E \\
Z
\end{array}\right]
$$

sendo $Z \in \mathbb{R}^{(n+1-m) \times g}$ uma matriz nula. Assim, obteremos:

$$
\begin{array}{ll}
\text { Minimize } & \frac{\beta}{2}\left(g^{T} Q g+{\overline{c_{q}}}^{T} g\right)+\frac{\alpha}{2}\left(\bar{x}^{T} \bar{R} \bar{x}+{\overline{c_{r}}}^{T} \bar{x}\right) \\
\text { Sujeito a } & -\bar{E} g+\bar{B} \bar{x}=b \\
& g+v=g_{u} \\
& \bar{x}+u=\overline{x_{u}} \\
& (g, \bar{x}, v, u) \geq 0 .
\end{array}
$$

Por simplicidade, eliminaremos as barras sobre as variáveis ao longo do texto.

\section{Problema Estocástico de Dois Estágios com Recurso Fixo para o FPO}

Nesse modelo, consideramos a geração das hidrelétricas como variáveis de primeiro estágio, $g_{1 i}$, enquanto a geração das termelétricas são variáveis de segundo estágio, $g_{2 i j}$. O vetor de probabilidade $a_{i j}=\left(a_{i 1}, a_{i 2}, a_{i 3}, \cdots, a_{i N}\right)$, tal que $N$ corresponde ao número de cenários considerados e $i$ indica o horário do planejamento $P$. Além disso, a matriz diagonal $M$ e o vetor $c_{m}$ correspondem aos custos de geração das termelétricas. A matriz $F$ será formada por colunas da matriz identidade, que representam os geradores das termelétricas. O vetor $g_{h}$ representa a meta diária das usinas hidrelétricas.

$$
\begin{array}{ll}
\text { Min } & \sum_{i=1}^{P}\left\{\frac{\beta_{i}}{2}\left(g_{1 i}^{T} Q g_{1 i}+c_{q}^{T} g_{1 i}\right)+\sum_{j=1}^{N} a_{i j}\left[\frac{\xi_{i}}{2}\left(g_{2 i j}^{T} M g_{2 i j}+c_{m}{ }^{T} g_{2 i j}\right)+\right.\right. \\
& \left.\left.+\frac{\alpha_{i}}{2}\left(x_{i j}^{T} R x_{i j}+c_{r}^{T} x_{i j}\right)\right]\right\} \\
\text { S. a } & \sum_{i=1}^{P} g_{1 i}=g_{h} ; \quad-E g_{1 i}-F g_{2 i j}+B x_{i j}=b_{i j} ; \\
& g_{1 i}+v_{1 i}=g_{u} ; \quad g_{2 i j}+v_{2 i j}=g_{2 u} ; \\
& x_{i j}+u_{i j}=x_{u} ; \quad\left(g_{1 i}, g_{2 i j}, x_{i j}, v_{1 i}, v_{2 i j}, u_{i j}\right) \geq 0 .
\end{array}
$$

O problema (6), é chamado também de Problema de Recurso (RP), cuja matriz de restrições possui $n_{t}=2 P\left[g_{1}+N\left(g_{2}+n+1\right)\right]$ colunas e $m_{t}=g_{1}+P\left[g_{1}+N\left(g_{2}+2 n+2\right)\right]$ linhas.

\subsection{Valor da Solução Estocástica}

Definition 3.1. A solução do RP é o valor que minimiza a função objetivo considerando o valor esperado da solução de segundo estágio, ou seja, ela determina a melhor solução de primeiro estágio 
considerando todos os cenários possíveis,

$$
R P=\min _{x} E_{\xi}[z(x, \xi)]
$$

Definition 3.2. A solução do problema de Valor Esperado (EV) considera o valor esperado da variável aleatória $\xi$,

$$
E V=\min _{x} z(x, \bar{\xi})
$$

Definition 3.3. O Resultado Esperado usando a solução do problema de Valor Esperado (EEV) é obtido da seguinte forma: considere $\bar{x}(\bar{\xi})$ a solução de $E V$; em seguida, resolva o problema de dois estágios assumindo $x=\bar{x}(\bar{\xi})$, ou seja, para cada cenário, apenas as variáveis de segundo estágio são otimizadas, pois as variáveis de primeiro estágio foram fixadas a priori; por fim, calcula-se o valor esperado da função objetivo,

$$
E E V=E_{\xi}[z(\bar{x}(\bar{\xi}), \xi]
$$

Definition 3.4. O Valor da Solução Estocástica (VSS) é definido como a diferença entre os valores EEV e RP. Assim, o VSS determina o ganho obtido pela solução estocástica ou a perda por desconsiderar a incerteza na tomada de decisão,

$$
V S S=E E V-R P .
$$

Proposition 3.1. Todo problema de programação estocástica de dois estágios satisfaz a desigualdade

$$
R P \leq E E V
$$

Proposition 3.2. Seja $f$ uma função densidade de probabilidade em $[a, b]$. Então,

$$
\sum_{i=1}^{N} t_{i-1} p_{i} \leq E[\phi(X)] \leq \sum_{i=1}^{N} t_{i} p_{i}
$$

desde que $E[\phi(X)]=\int_{a}^{b} t f(t) d t, p_{i}=P\left(t_{i-1} \leq X \leq t_{i}\right)=\int_{t_{i-1}}^{t_{i}} f(t) d t$ e $N$ é o número de pontos da partição.

Definition 3.5. Seja $\bar{P}$ uma restrição da distribuição de probabilidade $P$ ao intervalo $[a, b]$, ou seja, $\bar{f}:[a, b] \longrightarrow \mathbb{R}$, tal que $\bar{P}\left(t_{i-1} \leq \bar{X} \leq t_{i}\right)=P\left(t_{i-1} \leq X \leq t_{i}\right)+\epsilon$. A constante $\epsilon$ representa a n-ésima parcela do erro, sendo definida por $\epsilon=[1-P(a \leq X \leq b)] / n$.

\subsection{Teste de Shapiro-Wilk}

O teste de Shapiro-Wilk é um teste de normalidade cuja hipótese nula, $H_{0}$, considera que a amostra provém de uma distribuição normal, e a hipótese alternativa, $H_{1}$, corresponde ao caso contrário. Para realizar o teste de Shapiro-Wilk usaremos o software $R$, o qual calcula $W_{o}$ e o $p$ - valor. Assim, dado que o $p$-valor baixo é evidência contra $H_{0}$, então, para concluirmos se uma dada variável aleatória provém de uma população com distribuição normal, verificaremos duas condições: $W_{o}>W_{\alpha, n}$ e $p-$ valor $>\alpha$.

\subsection{Desigualdade de Jensen}

Esta desigualdade é baseada na relação entre a reta tangente e uma função convexa. Assim, definindo $c_{11}=\int_{a}^{\mu_{0}} d F(t)>0, c_{12}=1-c_{11}>0, \mu_{11}=\frac{1}{c_{11}} \int_{a}^{\mu_{0}} t d F(t), \mu_{12}=\frac{1}{c_{12}} \int_{\mu_{0}}^{b} t d F(t) \mathrm{e}$ $\phi\left(\mu_{0}\right)=J_{0}$, obteremos:

$$
J_{0} \leq J_{1} \equiv c_{11} \phi\left(\mu_{11}\right)+c_{12} \phi\left(\mu_{12}\right) \leq E[\phi(t)],
$$

que é um limite inferior para a solução estocástica $R P$. 


\subsection{Desigualdade de Edmundson-Madansky}

Um limitante superior para solução estocástica $R P$ é obtido pela desigualdade de EdmundsonMadansky. Esta desigualdade é motivada pela propriedade de um segmento de reta que liga dois pontos de uma função convexa. Seja $\mu_{0}=E(X)$, então

$$
M_{0}=\frac{\left(b-\mu_{0}\right)}{b-a} \phi(a)+\frac{\left(\mu_{0}-a\right)}{b-a} \phi(b) \geq E(\phi(X)) .
$$

Theorem 3.1. Sejam $\phi$ uma função convexa contínua em $[a, b]$ e $E(X)$ finita. Então $E[\phi(X)] e ́$ finito e $J^{n} \rightarrow E[\phi(X)] \leftarrow M^{n}$. Assumindo que cada subintervalo torna-se arbitrariamente pequeno quando $n$ tende ao infinito [5].

\section{Aplicação}

Nos testes numéricos, definimos uma decomposição da capacidade de geração de potência ativa da seguinte forma: 2/3 da capacidade de geração padrão correspondendo às usinas hidrelétricas e o restante da capacidade de geração às usinas termelétricas. O limite inferior dos geradores sendo nulo para hidrelétricas e termelétricas. O limite superior do fluxo de potência ativa igual a capacidade total de geração padrão, e o limite inferior igual ao negativo do limite superior. Os custos de geração de potência ativa das usinas termelétricas sendo cinquenta vezes maior que os custos de geração das usinas hidrelétricas. O vetor de probabilidade foi obtido, como na Definição 3.5, usando o histórico de consumo disponibilizado pelo Operador Nacional do Sistema Elétrico Brasileiro. Escolhemos o dia 01 de outubro de 2020 como referência de estudo. Assim, coletamos os dados do consumo energético por hora, de 25 a 30 de setembro de 2020, e verificamos que os dados provêm de uma distribuição normal.

\subsection{IEEE30}

Este problema representa uma aproximação do Sistema de Potência Elétrica Americano, no Meio-Oeste dos EUA. A capacidade de geração é de $280 \mathrm{MW}$, com 6 geradores. Assim, definimos $g_{1}=4$ e $g_{2}=2$. Como o número de linhas de transmissão é 41 , a matriz de restrições do $R P$ terá dimensões $A(97+2094 N, 192+2112 N)$.



Figura 1: Fluxo de Potência Ótimo por hora.

* Os valores do eixo vertical são da ordem de $10^{5} \mathrm{um}$ 
Na Figura 1, observamos a variação diária das soluções $E V, R P$ e $E E V$, juntamente com a solução $R E A L$ que corresponde à solução do FPO com o consumo do dia de referência. Ressaltamos que $R P \leq E E V$ em todos os horários. Isso verifica a Proposição 11 e garante que $V S S \geq 0$. Portanto, seria vantajoso considerar a solução estocástica no FPO.

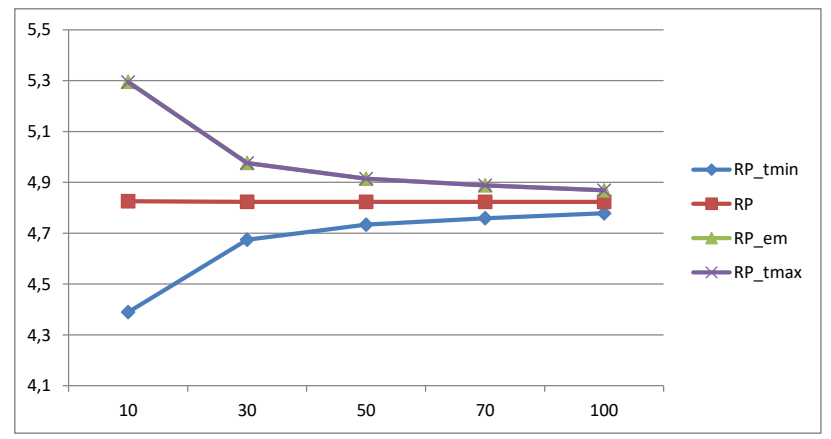

Figura 2: Solução do Problema de Recurso, com $P=24$ horas, versus o Número de Cenários.

* Os valores do eixo vertical são da ordem de $10^{6} \mathrm{um}$.

Na Figura 2, comparamos nossa proposta de solução, baseada nas propriedades de soma inferior, superior e de Riemann, com aproximações dos limites inferior de Jensen e superior de EdmundsonMadansky. O melhor desempenho que temos observado é o da solução $R P$. Nesta solução, o $t_{i}^{*}$ é igual ao ponto médio de cada intervalo $\left[t_{i-1}, t_{i}\right]$ da partição. Se, na Desigualdade 13 , substituirmos $c_{i}=\overline{p_{i}}$ e $\mu_{i}=t_{i}^{*}$, então, $R P$ seria uma aproximação do limite inferior de Jensen.

Tabela 1: Valor Ótimo do Problema 6

\begin{tabular}{cccc}
\hline & Valor ótimo $\left(10^{6} \mathrm{um}\right)$ & Tempo de CPU $(\mathrm{s})$ & Número de iterações \\
\hline$F_{R P D}$ & 4.8231 & 81.7873 & 63 \\
$F_{R E A L}$ & 4.9831 & 0.9697 & 60 \\
\hline
\end{tabular}

Na Tabela 1, observamos a proximidade entre os valores $F_{R P D}$ e $F_{R E A L}$, ou seja, o Prédespacho do FPO obtido pela solução estocástica representa 96,79\% do valor ótimo do problema com o consumo verificado no dia de referência. Acreditamos que o alto tempo de CPU na resolução do Problema de Recurso seja devido ao número de cenários considerados, $N=100$.

\section{Conclusões}

De acordo com os testes numéricos, o modelo proposto apresentou resultados coerentes. Isso resulta da aproximação da distribuição de probabilidade proposta e, também, da solução $R P$ escolhida. Conforme observamos na Figura 1, o Valor da Solução Estocástica foi positivo em todos os horários, portanto, seria lucrativo considerar a solução estocástica em vez da solução determinística. 


\section{Agradecimentos}

Agradecemos à Coordenação de Aperfeiçoamento de Pessoal de Nível Superior (CAPES) e ao Conselho Nacional de Desenvolvimento Científico e Tecnológico ( $\mathrm{CNPq}$ ) pelo apoio e aporte financeiro no desenvolvimento deste trabalho.

\section{Referências}

[1] Birge, J. R. The Value of the Stochastic Solution in Stochastic Linear Programs with Fixed Recourse, Mathematical Programming, vol. 24, pp. 314-325, 1982.

[2] Carvalho, M. F., Soares, S. and Ohishi, T. Optimal Active Power Dispatch by Network Flow Approach. IEEE Transactions on Power Systems, vol. 3, no. 3, pp. 1640-1647, 1988.

[3] Dantzig, G. B. Linear Programming Under Uncertainty, Management Science, vol. 50, no. 12 Supplement, pp. 1764-1769, Dec. 2004.

[4] Gondzio, J. Interior Point Methods 25 Years Later, European Journal of Operational Research, vol. 218 , pp. 587-601, 2012.

[5] Huang, C. C., Ziemba, W. T. and Ben-Tal, A. Bounds on the Expectation of a Convex Function of a Random Variable: With Applications to Stochastic Programming. Operations Research, vol. 25, no. 2, pp. 315-325, 1977.

[6] Karmarkar, N. A New Polynomial-Time Algorithm for Linear Programming, Combinatorica, vol. 4, no. 4, pp. 373-395, 1984.

[7] Kojima, M., Mizuno, S. and Yoshise A. A Primal-dual Interior-Point Method for Linear Programming, Progress in Mathematical Programming, Interior-Point and Related Methods, Spring-Verlag, New York, pp. 29-47, 1989.

[8] Momoh, J. A., El-Hawary, M. E. and Adapa, R. A Review of Selected Optimal Power Flow Literature to 1993, Part I: NonLinear and Quadratic Programming Approaches. IEEE Transactions on Power Systems, vol. 14, no. 01, pp. 96-104, Feb. 1999.

[9] Oliveira, A. R. L., Soares, S. and Nepomuceno, L. Optimal Active Power Dispatch Combining Network Flow and Interior Point Approaches, IEEE Transactions on Power Systems, vol. 18, no. 4, pp. 1235-1240, Nov. 2003.

[10] Quintana, V. H., Torres, G. L. and Medina-Palomo, J. Interior Point Methods and their Applications to Power Systems: A Classification of Publications and Software Codes, IEEE Transactions on Power Systems, vol. 15, no. 1, pp. 170-176, 2000.

[11] Soroudi, A. and Amraee T. Decision making under uncertainty in energy systems: State of the art. Renewable and Sustainable Energy Reviews, vol. 28, pp. 376-384, 2013.

[12] Vanderbei, R. J. Linear Programming - Foundations and Extensions, 2th Edition, Kluwer Academics Publishers, Boston, USA, 2001. 